\title{
Android Based Home-Automation using Microcontroller
}

\author{
Dr. Harsh Dev \\ Dean, Pranveer Singh Institute of Technology, kanpur \\ Email: drharshdev@gmail.com \\ Anjani Gupta \\ Department of Computer Science and Engineering, Pranveer Singh Institute of Technology, kanpur \\ Email: anjani050296@gmail.com \\ Himanshi Jain \\ Department of Computer Science and Engineering, Pranveer Singh Institute of Technology, kanpur \\ Email: himanshijain95hj@gmail.com \\ Aditya Agarwal \\ Department of Computer Science and Engineering, Pranveer Singh Institute of Technology, kanpur \\ Email: agarwaladitya0502@gmail.com \\ Raghvendra Gupta \\ Department of Computer Science and Engineering, Pranveer Singh Institute of Technology, kanpur \\ Email: raghu1996gupta@gmail.com \\ Kavleen Kaur \\ Department of Computer Science and Engineering, Pranveer Singh Institute of Technology, kanpur \\ Email: kavleenbehr@gmail.com
}

\begin{abstract}
Due to the availability of complicated devices in industry, models for consumers at lower cost of resources are developed. Home Automation systems have been developed by several researchers. The limitations of home automation includes complexity in architecture, higher costs of the equipment, interface inflexibility. In this paper as we have proposed, the working protocol of PIC 16F72 technology is which is secure, cost efficient, flexible that leads to the development of efficient home automation systems. The system is operational to control various home appliances like fans, Bulbs, Tube light. The following paper describes about components used and working of all components connected. The home automation system makes use of Android app entitled "Home App" which gives flexibility and easy to use GUI.
\end{abstract}

Keywords - Android Application, BC547 Transistor, PIC Microcontroller, Relays.

\section{INTRODUCTION}

IOT is Internet of Things which basically denotes the intercommunication among devices by using embedded system [1]. The backbone of the Smart Homes is Home Automation which means controlling all the appliances without going close to the switches to control them. Home Automation has become cost effective using embedded systems. User comfort, security and flexibility along with maintaining living standard is provided by embedded system. The Home Automation comprises of controllers viz. server of the system.

\section{METHODOLOGY}

There are two sections in which the model is divided i.e. security and automation.

For security all the vigorous attempts are made to make the system secured. For the automation of the system all the procedure and methods of operating is handled [2].

The owner of the house has the authority over all the home appliances. The user has the default password. As the user login the app, an OTP is send to the registered number. This OTP is then valid for only ten seconds.
For the user login module, we have introduced user id as well as password for the security purpose in the android application. The mobile is connected via hotspot to the laptop.

The android application "Home App" sends request to the server database and database passes on the request to the hardware connected to the laptop through UART driver of PIC 16F72 microcontroller. The "Home App" and server as well as server and laptop is a two-way communication whereas the communication between hardware and laptop is one way or serial communication. HTTP and RS232 are following protocols which we are using for communication 


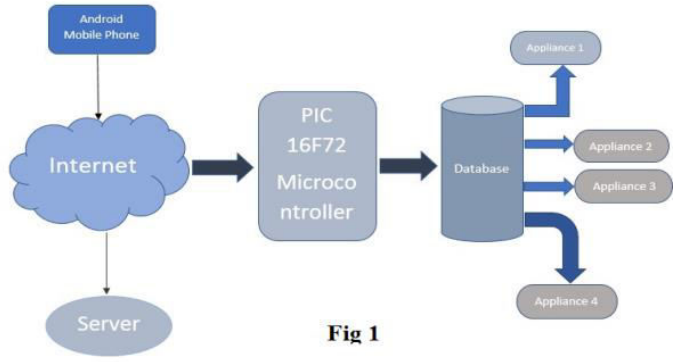

\subsection{Software Requirements}

1.1.1 Android

The android application developed "Home App" is certainly based on Linux operating system which uses Java and XML- languages for running applications and this provides flexible control of devices. The main purpose of this project is using Wi-Fi Hotspot to send control signals from mobile to hardware [11].

\subsubsection{XAMPP}

In "Home App" Xampp is used to connect SQL server for database for turning on / off the statuses of the appliances connected.

\subsubsection{Embedded C}

It is a language which is not only used in microcontrollers but also to define common issues prevailing among $\mathrm{C}$ extensions.

\subsection{Hardware Requirements}

\subsubsection{PIC Microcontroller}

The PIC 16F72 microcontroller contains UART driver which is used to connect the hardware with the laptop. To offload the I/O tasks from CPU, PIC 16F72 is used.

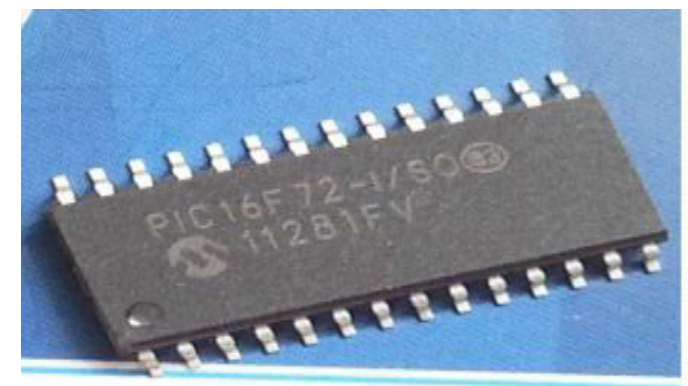

Fig 2

\subsubsection{Relay}

Relay is used for switching ON/OFF the device or to vary the light brightness. Relays are associated with every appliance used in the system. Other part or the end of the relay is connected to PIC16F72 microcontroller board [1].

1.2.3 USB Keyboard and mouse

1.2.4 HDMI monitor and cable

1.2.5 Micro USB power adapter (smart phone charger)

\section{DESIGN OVERVIEW}

The design of the proposed model is shown through Use case Diagram and Entity Relationship diagram [11].

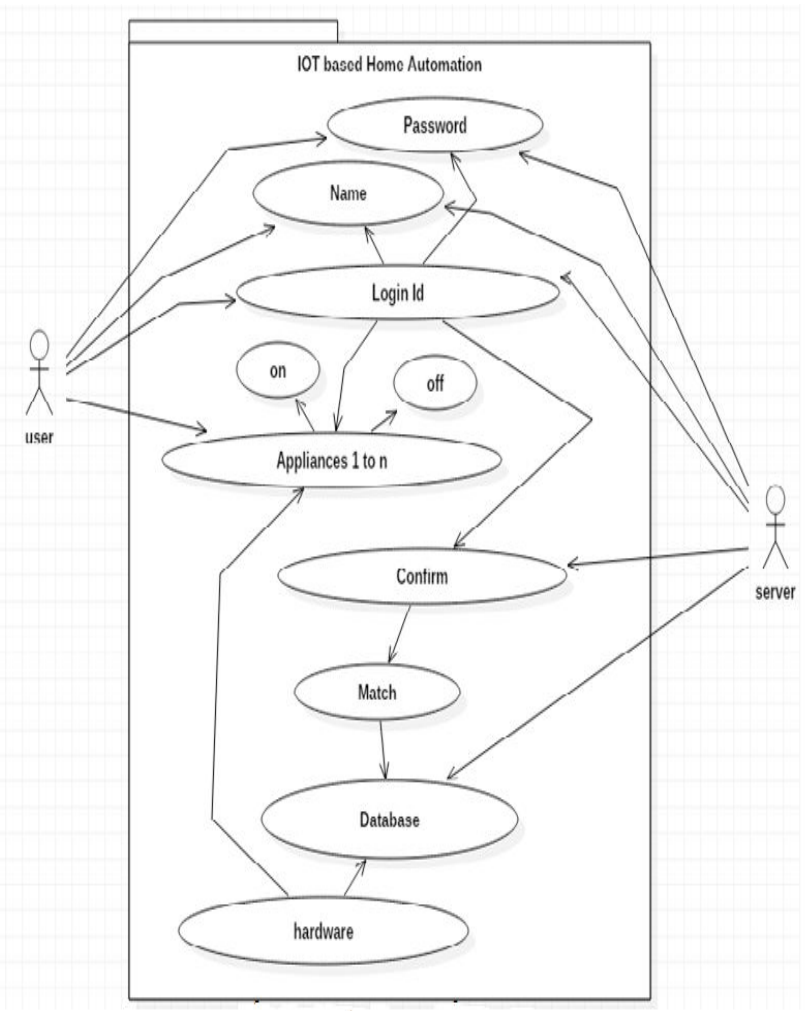

Fig 3. Use Case diagram 


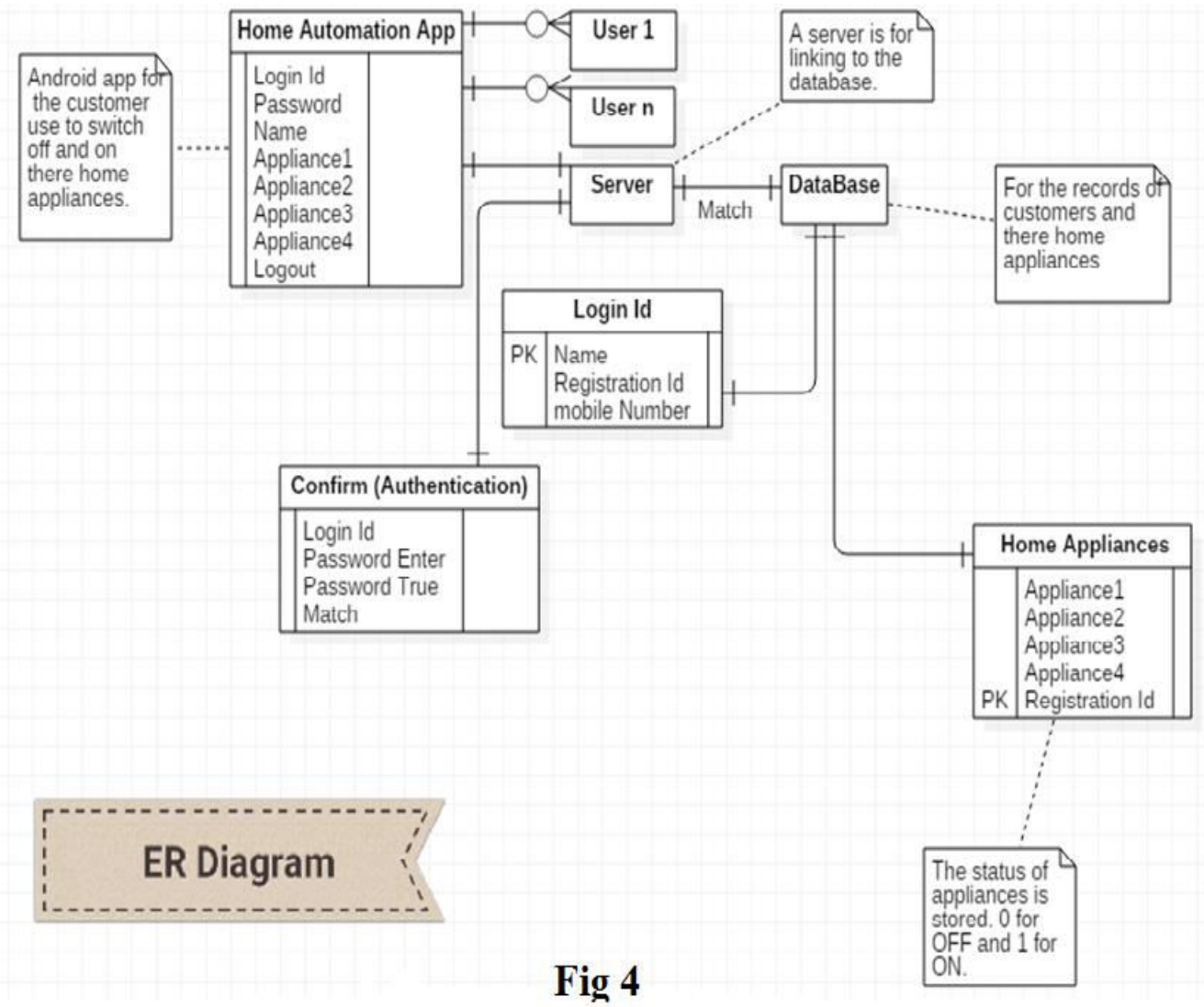

\section{IMPLEMENTATION AND EXPERIMENTATION}

The implementation here depicts the status of the hardware in ON/OFF state [4]. Firstly we login into the app "Home App" fig 5(a). This username and password gets saved in admin database. Now the user has control over all their home appliance and he can switch ON/OFF as per his needs.

In the "Home App", the user has to login everytime he opens it because due to security reasons.

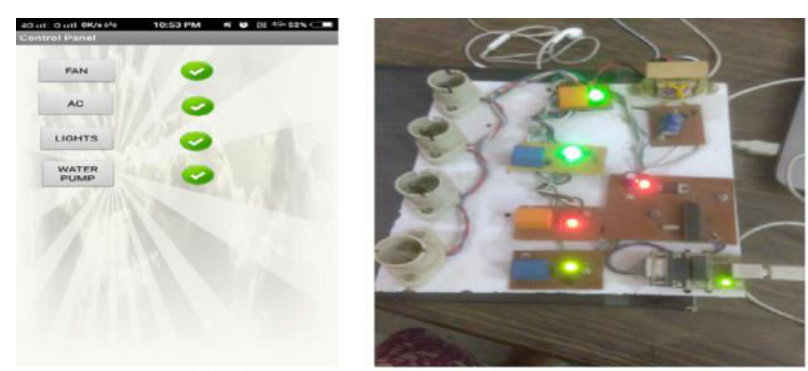

Fig 5(b). Status of devices in ON state

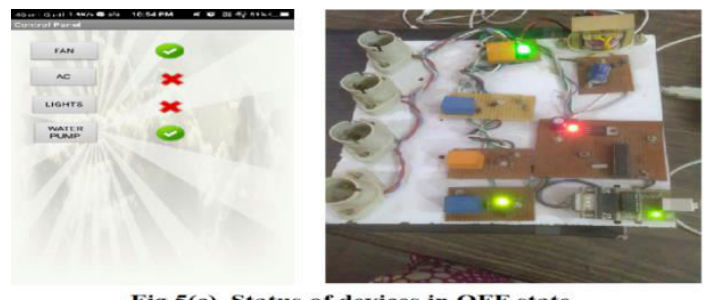

Fig 5(c). Status of devices in OFF state

\section{RESULTS AND DISCUSSIONS}

The paper offers frugal, safe, frequently accessible results for home automation which consists of more features and it is very cost effective as compared to others. The proposal discussed in this paper is new and it has attained the objective of controlling home appliances through hotspot without going near to the switches. The program 
coded in embedded $\mathrm{C}$ in the PIC microcontroller has been used for switching $\mathrm{ON}$ and OFF the following four appliances. When the user switches $\mathrm{ON}$ in the android app appliance1, then that appliance1 switches ON through the microcontroller. Likewise, other appliances also work same. The project is working efficiently in all tests.

A conclusion section must be included and should indicate clearly the advantages, limitations, and possible applications of the paper. Although a conclusion may review the main points of the paper, do not replicate the abstract as the conclusion. A conclusion might elaborate on the importance of the work or suggest applications and extensions.

\section{CONCLUSION}

In this paper as we have proposed, the working protocol of PIC 16F72 technology which is secure, cost efficient, flexible and $\mathrm{Wi}-\mathrm{Fi}$ based, providing solution to home automation by using android smart "Home App". The system will expedite users to pair with any sort of android devices through $\mathrm{Wi}-\mathrm{Fi}$ and control the electrical appliances of the room.

The "Home App" developed is easy to operate providing flexibility and rich GUI interface to the users.

\section{FUTURE SCOPE}

We have introduced the recommended system which is being reinforced via aid of Arduino/AVR/ARM based microcontroller technology. The same work can be extended by using advanced wireless communication such as ZigBee to increase the range of communication. By the use of Smart IR sensor technology, we can save electricity as well as the environment. The application can also be extended for platforms other than Android like IOS and Windows Phones. The application would then be more eco-friendly.

\section{ACKNOWLEDGEMENTS}

Authors are gratitude to Head and dept. of CSE, staff members and college management for their sincere and financial support for carrying out this research work on Internet of Things (IOT).

\section{REFERENCES}

\section{Journal Papers:}

[1] Vimal Nakrani, Internet of things (IOT) based smart home automation, International Journal of Recent Trends in Engineering \& Research (IJRTER), vol. 3, pp. 231-236, March - 2017.

[2] Viraj Mali, Home automation and security using arduino microcontroller, International Journal of Research in Advent Technology Special Issue National Conference, pp. 214-217, 19 March 2016.

[3] A. Mathi, Home automation based on IOT, International Journal of Advance Research, Ideas and Innovations in Technology, vol. 3, pp. 1556-1559, 2017.

[4] Waiz Khan, Smart home (home automation), International Journal of Latest Transactions in
Engineering and Science, vol. 2, pp. 22-27, April 2017.

[5] Mohamed Abd El-Latif Mowad, Smart home automated control system using android application and microcontroller, International Journal of Scientific \& Engineering Research, vol. 5, pp. 935939, May-2014.

[6] Abhishek Bhat, Home automation using internet of things , International Research Journal of Engineering and Technology (IRJET), vol. 4, pp. 917920, July -2017.

[7] Abhishek Saxena, Smart home automation using android application, International Journal of Scientific Research and Management Studies (IJSRMS), vol. 3, pp. 326-329.

[8] M.Abivandhana, Smart home automation based on IOT and android technology, International Journal of Engineering Science and Computing, vol. 7, pp. 59435946, March 2017.

[9] https://hacktronics.co.in/microcontrollers/pic16f72iso-microchip-8-bit-pic-microcontroller

\section{Proceedings Papers:}

[10] Kim Baraka, Low cost arduino/android-based energyefficient home automation system with smart task scheduling, Fifth International Conference on Computational Intelligence, Communication Systems and Networks, pp. 296, 2013.

[11]Er. Vikram Puri, Real time smart home automation based on PIC microcontroller, bluetooth and android technology, International Conference on Computing for Sustainable Global Development (INDIACom), pp. 1478-1484, 2016.

\section{Biographies and Photographs}

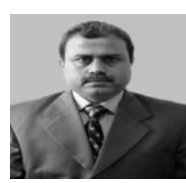

Dr. Harsh Dev is a Professor in Dept. of Computer Science and Engineering, he is also holding a position of Dean Research at Pranveer Singh Institute of technology, Kanpur, India. He got his M.Sc. Degree in 1995 from Lucknow University, India \& Ph.D. degree in Computer Science in 2005 from Babasaheb Bhimrao Ambedkar University, Lucknow, India.

He has 23 years of teaching experience and 16 years of research experience in the field of Computer Graphics, Software Engineering and Data Mining. He has published more than 40 International and National publications. He is a member of Computer Society of India, Indian Science Congress and International Association of Engineers (IAENG).

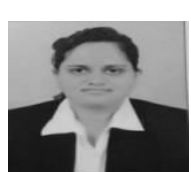

Anjani Gupta is a Student pursuing B.Tech (2014-2018) in Dept. of Computer Science and Engineering, Pranveer Singh Institute of Technology.

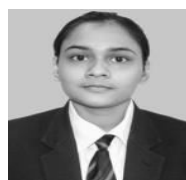

Himanshi Jain is a Student pursuing B.Tech (2014-2018) in Dept. of Computer Science and Engineering, Pranveer Singh Institute of Technology. 